\title{
Crystal optics for hard-X-ray spectroscopy of highly charged ions ${ }^{\text {is }}$
}

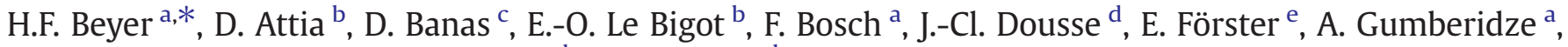 \\ S. Hagmann ${ }^{\text {a }}$, S. Heß ${ }^{\text {a }}$, J. Hoszowska ${ }^{\text {d }, ~ P . ~ I n d e l i c a t o ~}{ }^{\text {b }}$, P. Jagodzinski ${ }^{c}$, Chr. Kozhuharov ${ }^{\text {a }}$, Th. Krings ${ }^{g}$, \\ D. Liesen ${ }^{a}$, X. Ma ${ }^{\text {h }}$, B. Manil ${ }^{\mathrm{i}}$, I. Mohos ${ }^{\mathrm{g}}$, M. Pajek ${ }^{\mathrm{c}}$, D. Protić ${ }^{\mathrm{g}}$, R. Reuschl $^{\mathrm{a}}$, J. Rzadkiewicz ${ }^{\mathrm{j}}$, A. Simionovici ${ }^{\mathrm{k}}$, \\ U. Spillmann ${ }^{\text {a }}$, Z. Stachura ${ }^{1}$, Th. Stöhlker ${ }^{a}$, M. Trassinelli ${ }^{\mathrm{m}}$, S. Trotsenko ${ }^{\text {a }}$, A. Warczak ${ }^{\mathrm{n}}$, \\ O. Wehrhan ${ }^{\text {e, E. Ziegler }}{ }^{\mathrm{f}}$
}

a GSI, Planckstraße 1, D-64291 Darmstadt, Germany

${ }^{\mathrm{b}}$ Laboratoire Kastler Brossel, Ecole Normale Supérieure, CNRS et Université Pierre et Marie Curie-Paris 6, Case 74, 4 place Jussieu, F-75005 Paris, France

c Institute of Physics, Swietokrzyska Academy, 25-406 Kielce, Poland

d Department of Physics, University of Fribourg, Chemin du Musée 3, CH-1700 Fribourg, Switzerland

e Institut für Optik und Quantenelektronik, Friedrich Schiller-Universität, D-07743 Jena, Germany

${ }^{\mathrm{f}}$ ESRF, F-38043 Grenoble, France

g FZ Jülich, Institut für Kernphysik, D-52425 Jülich, Germany

${ }^{\mathrm{h}}$ Institute of Modern Physics, 730000 Lanzhou, China

${ }^{\mathrm{i}}$ CIRIL-GANIL, rue Claude Bloche, F-14070 Caen, France

${ }^{\mathrm{j}}$ Andrzej Soltan Institute for Nuclear Studies, 05-400 Swierk, Poland

${ }^{k}$ LGIT, Observatoire des Sciences de l'Univers de Grenoble, F-38041 Grenoble Cedex 9, France

${ }^{1}$ Institute of Nuclear Physics, PL-31-342, Cracow, Poland

${ }^{\mathrm{m}}$ Institut des Nanosciences de Paris, Campus Boucicaut, 140 rue de Lourmel, F-75015 Paris, France

${ }^{n}$ Institute of Physics, Jagiellonian University, PL-30059 Cracow, Poland

\section{Introduction}

The motivation for the present development is the interest in highly charged ions with only one or few electrons as a sensitive probe of relativistic and quantum-electrodynamic (QED) effects in the hitherto unexplored domain of very strong electromagnetic fields [1]. Such ions

is This paper was presented at the 19th "International Congress on X-ray Optics and Microanalysis" (ICXOM-19) held in Kyoto (Japan), 16-21 September 2007

\footnotetext{
* Corresponding author.

E-mail address: h.beyer@gsi.de (H.F. Beyer).
}

\begin{abstract}
A twin crystal-spectrometer assembly, operated in the focusing compensated asymmetric Laue geometry has to to the focusing crystal optics is a specially developed two-dimensional position-sensitive X-ray detector optics and $\mathrm{X}$-ray sources. Results are reported from several tests employing a ${ }^{169} \mathrm{Yb}$ gamma-ray source and the Lyman radiation of one-electron $\mathrm{Pb}^{81+}$ ions traveling at a velocity corresponding to $\beta \equiv v / c \approx 0.59$. The features of the instrument presented may be useful in many applications where it appears difficult to make the leap from conventional X-ray energy measurements to wavelength-dispersive spectroscopy based on crystal optics.
\end{abstract}

like completely stripped $\mathrm{Au}^{79+}, \mathrm{Pb}^{82+}$ or $\mathrm{U}^{92+}$ are available in the experimental storage ring ESR at GSI Darmstadt. QED contributions to the ground-state binding energy in one-electron systems can be determined from an accurate spectroscopy of the corresponding $K$-shell transitions which give rise to the emission of hard X-rays. Previously this X-ray source has been studied with conventional solid state $\mathrm{Ge}(\mathrm{i})$ detectors but this technique is facing its limits because of insufficient spectral resolution. The transition to wavelength-dispersive X-ray spectroscopy using crystal optics is a challenging task because of its inherently low detection efficiency. The way out is a compromise trading off some of the crystals' superior resolution for increased detection efficiency.

For the present exercise we went through a careful optimization which might also serve as an example for other applications. We employ the 
focusing compensated asymmetric Laue (FOCAL) case making use of a focusing crystal plus an enhanced integrated reflectivity by a corresponding broadening of the rocking curve compatible with the natural line width of the radiation under study. A further complication is given by the fact that the X-rays are emitted by ions that travel at a high velocity. With the crystal optics chosen and the vertical orientation of the Rowland-circle plane, the spectral resolution can be preserved at velocities as high as 60\% of the speed of light $c$. Two spectrometers are installed to compensate for possible small fluctuations in the direction of the ion beam.

In the present implementation of FOCAL, a spectrum can be measured either by scanning a conventional $\mathrm{Ge}(\mathrm{i})$ detector with a narrow slit in the dispersive direction or using a position-sensitive Xray detector in order to make use of the multiplex advantage yielding an overall higher measurement speed. Preliminary results obtained with a one-dimensional position-sensitive detector have been communicated earlier [2]. To take full advantage of the FOCAL crystal optics a two-dimensional position-sensitive X-ray detector has been developed. Here we report on first results obtained with this new detector. The present studies are part of a common effort together with the development of low-temperature calorimetric detectors [3].

\section{The X-ray source}

The experiments were carried out at the ESR storage ring at GSI Darmstadt. A schematic picture of the ESR is shown in Fig. 1. This storage ring with all features of a synchrotron has a circumference of $108 \mathrm{~m}$. In one of the two long straight sections an electron cooler is installed and in the other one a gas-jet target can be operated. For the present experiment the ESR was periodically loaded with approximately $10^{8}$ bare $\mathrm{Pb}^{82+}$ ions at a velocity of $\beta \equiv v / c \approx 0.59$. X-rays are produced both in the electron cooler and in the gas target due to the decay of excited one-electron ions formed by electron-ion and by atom-ion interactions. For a more detailed account on the atomic processes as well as on the specific experimental techniques see, e.g., ref. [4]. For the present investigation we made use of the gas target as an X-ray source. The kind of X-rays produced there is strongly dependent on the target species and on the ion-beam velocity. To favor electron capture into excited states of the projectile a krypton gas jet was chosen for its high atomic number. Within a typical cycle time of about $2 \mathrm{~min}$ a high fraction exceeding 0.5 of the inventory of ions in the storage ring contributes to the collisional production of Lyman photons useful for spectroscopy.

Ions capable of emitting a Lyman photon have lost one unit of charge in passing the gas jet and are magnetically separated from the main ion beam in the next dipole magnet. The one-electron ions can be detected with a fast particle detector installed in a pocket of the dipole vacuum chamber. As indicated in Fig. 1 two FOCAL crystal spectrometers are symmetrically arranged at the gas-jet target viewing the same X-ray source defined by the small interaction volume of the ion beam and the gas jet. The diameter of a cooled ion beam is about $2 \mathrm{~mm}$ whereas the gas jet is about $5-10 \mathrm{~mm}$ in diameter. The X-rays detected can be measured in a delayed coincidence with the down-charged particles registered by the particle detector.

At an observation angle of $90^{\circ}$ the X-rays are red shifted with a laboratory wavelength

$\lambda_{\text {lab }}=\gamma \lambda_{\text {ion }}$,

where $\gamma=\left(1-\beta^{2}\right)^{-1 / 2}$ is the Lorentz factor and $\lambda_{\text {ion }}$ denotes the wavelength in the rest frame of the fast ion. Wavelength uncertainties introduced via the Lorentz transformation (1) depend on the angular and velocity uncertainties and can be severe. At $90^{\circ}$ observation we are in a geometry that is near its maximum angular sensitivity but at a minimum velocity sensitivity. For the $90^{\circ}$ observation, the relative wavelength uncertainties or shifts may be expressed as

$$
\left(\frac{\Delta \lambda}{\lambda}\right)_{\beta}=\beta \gamma^{2} \Delta \beta
$$

and

$$
\left(\frac{\Delta \lambda}{\lambda}\right)_{\theta}=\beta \Delta \theta
$$

where $\theta$ denotes the observation angle relative to the ion-beam direction. With a two-sided observation at angles $\theta$ and $\pi-\theta$, angular uncertainties cancel and the wavelength in the emitter system is given by the arithmetic mean of the two laboratory wavelengths

$\lambda_{\text {ion }}=\frac{\lambda_{\text {lab }}(\theta)+\lambda_{\text {lab }}(\pi-\theta)}{2 \gamma}$.

This is the reason for setting up a twin spectrometer as will be described in Section 4.1.

During the alignment of the spectrometer and for the online calibration gamma-ray lines of the radioactive isotope ${ }^{169} \mathrm{Yb}$ were used.

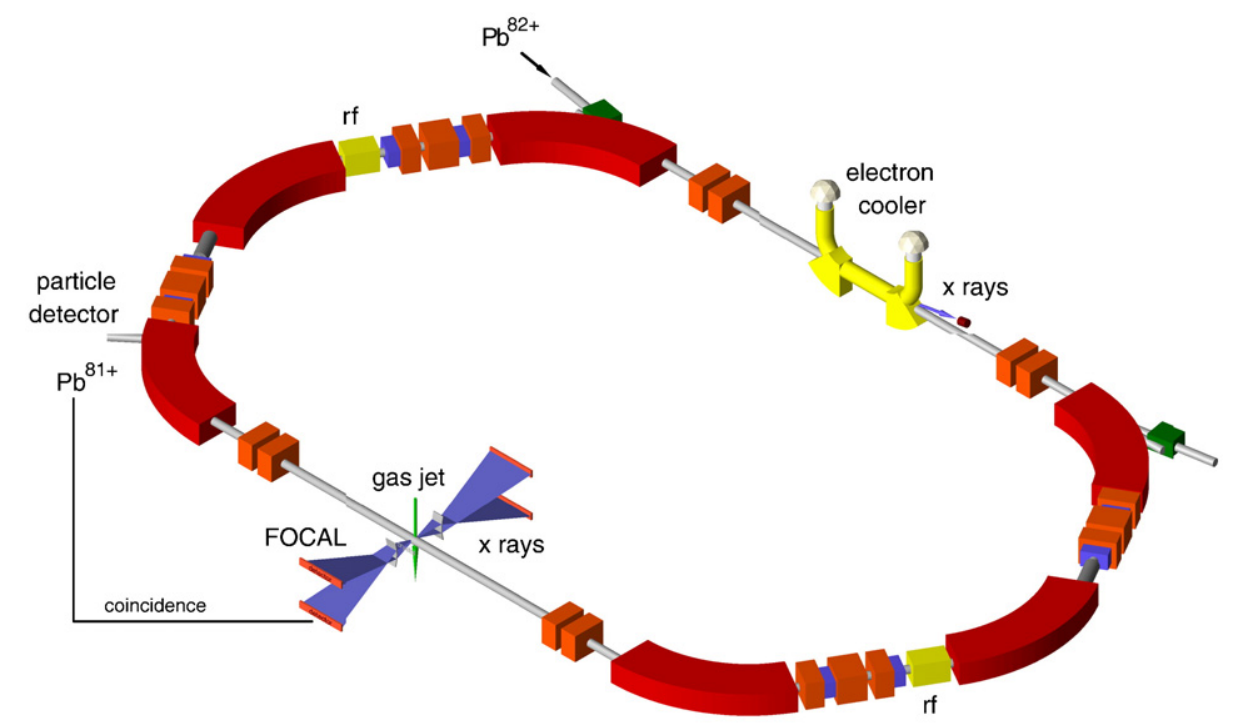

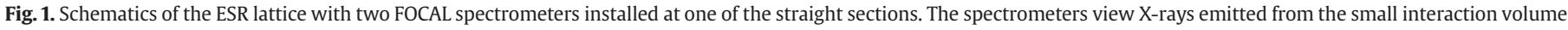
between ion beam and gas jet. 


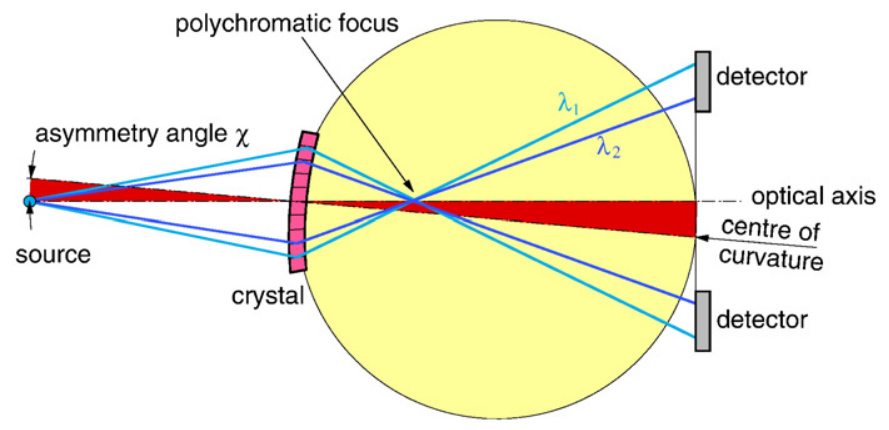

Fig. 2. Schematics of the FOcusing Compensated Asymmetric Laue optics, FOCAL.

The radioactive probes were produced by neutron activation of isotopically enriched samples of Ytterbium oxide containing $25 \mathrm{mg}$ of the ${ }^{168} \mathrm{Yb}$ isotope. The probes are small tablets of $5 \mathrm{~mm}$ diameter encapsulated in pure aluminum to approximately resemble the size of the fast-beam source. The calibration sources could be remotely positioned onto the optical axis of FOCAL halfway between the ion beam and the curved crystals of the crystal spectrometers.

\section{FOCAL crystal optics}

\subsection{X-ray optical scheme}

The principle of the X-ray-optical arrangement is shown in Fig. 2. A cylindrically curved Si crystal is used with its (220) planes deviating by an asymmetry angle $\chi=2^{\circ}$ from their perpendicular orientation relative to the principal crystal faces. In the figure the crystal is shown rotated by the asymmetry angle with the small X-ray source located on an axis that runs through the crystal's apex following the tilted lattice planes. This way two wavelength spectra appear symmetrically below and above the horizontal optical axis compensating for the asymmetry introduced by the use of an asymmetric crystal cut. A polychromatic line focus is formed whereas monochromatic focusing occurs near the periphery of the Rowland circle indicated. For small Bragg angles-in our case they are close to $3^{\circ}$-focusing errors remain small if the X-ray detectors are placed on a common vertical line as suggested in the figure. A more detailed geometrical description of the crystal optics together with analytical formulae and the numerical simulation procedure MacRay has been given earlier [5]

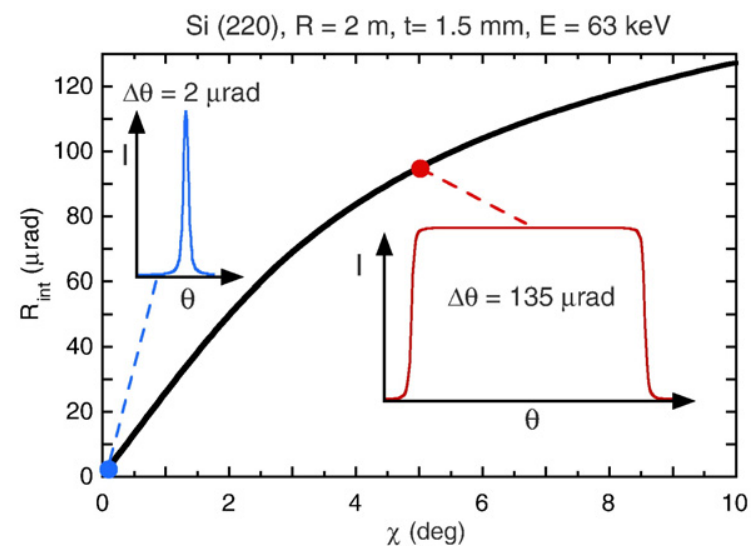

Fig. 3. Calculated integrated reflectivity for the focusing asymmetric Laue case as a function of the asymmetry angle. The example is for a $t=1.5 \mathrm{~mm}$-thick $\mathrm{Si}(220)$ crystal with a radius of curvature of $2 \mathrm{~m}$ and an X-ray energy of $63 \mathrm{keV}$. The rise of the integrated reflectivity with the asymmetry angle is accomplished by a corresponding broadening of the reflection curve as indicated by the two insets.

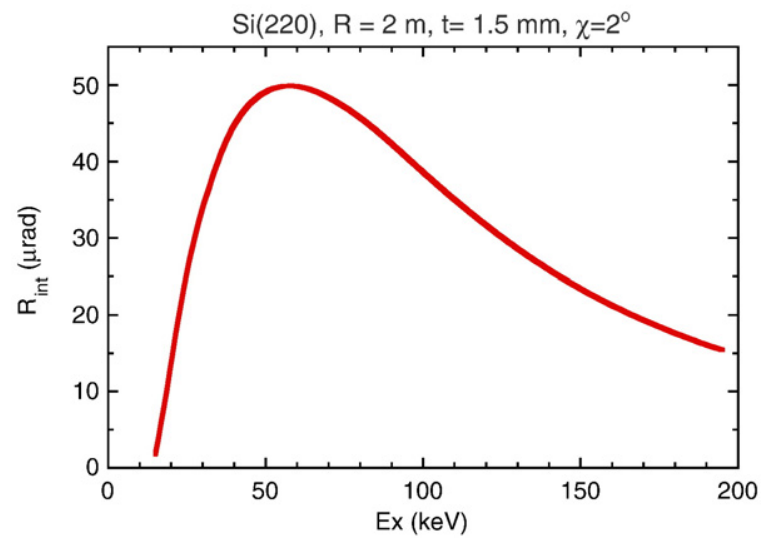

Fig. 4. Dependence of the integrated reflectivity on the X-ray energy.

\subsection{Parameter optimization}

The introduction of an asymmetric crystal cut leads to the well known broadening of the crystal rocking curve due to a combined elastic anisotropy and diffraction effect $[6,7,8]$.

Fig. 3 shows the increase of the calculated integrated reflectivity with the asymmetry angle $\chi$ for a $\mathrm{Si}(220)$ Laue crystal with a thickness of $1.5 \mathrm{~mm}$ and a radius of curvature of $R=2 \mathrm{~m}$ at an X-ray energy of $63 \mathrm{keV}$. For our present application we chose an asymmetry angle $\chi=2^{\circ}$ gaining an intensity boost of a factor of about 20 over the symmetric Laue case.

For the same crystal the calculated integrated reflectivity in dependence of the X-ray energy is displayed in Fig. 4. Using the $63 \mathrm{keV}$ gamma-ray line of ${ }^{169} \mathrm{Yb}$ as our main calibration line we are operating the spectrometer at an optimum energy, i.e. near the maximum of the curve displayed in Fig. 4 . The complete set of design parameters has already been compiled previously [2].

\section{Implementation}

\subsection{Bi-FOCAL}

The two FOCAL spectrometers already indicated in Fig. 1 are more clearly shown in Fig. 5. Both spectrometers are optically aligned as to

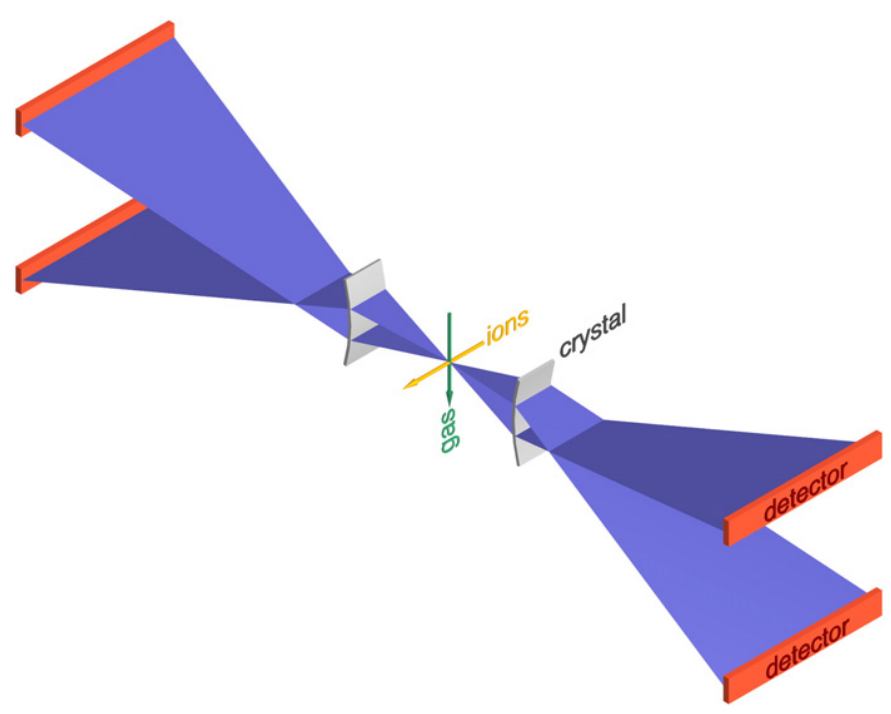

Fig. 5. Bi-FOCAL: two FOCAL spectrometers are symmetrically arranged around the ion beam viewing the common X-ray source realized by the small interaction volume between ion beam and gas jet. 


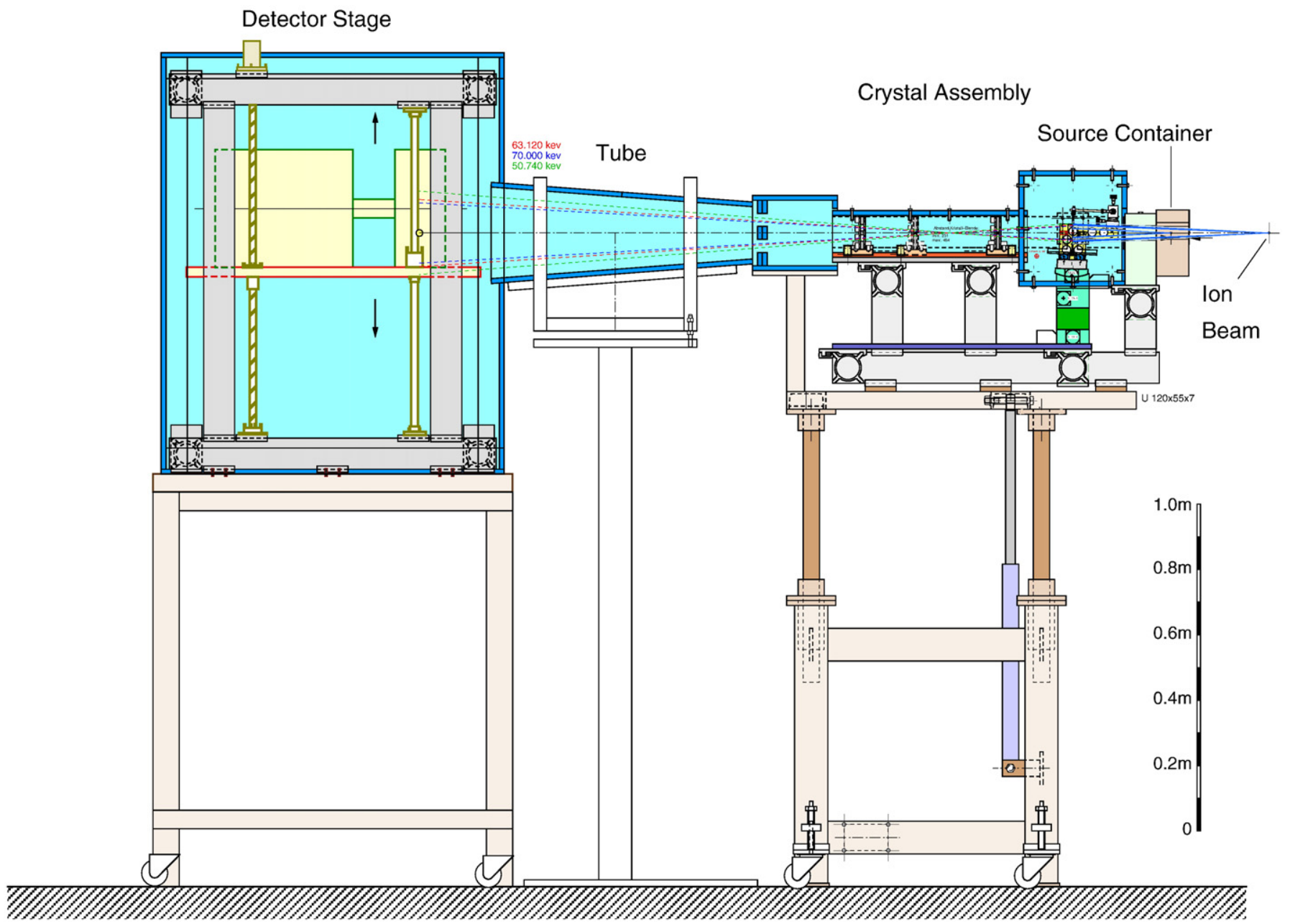

Fig. 6. Side view of one FOCAL spectrometer arranged in a modular design for convenient handling.

follow a common optical axis running through the intended centre of the interaction region and oriented perpendicularly to the gas-jet and to the ion-beam direction.

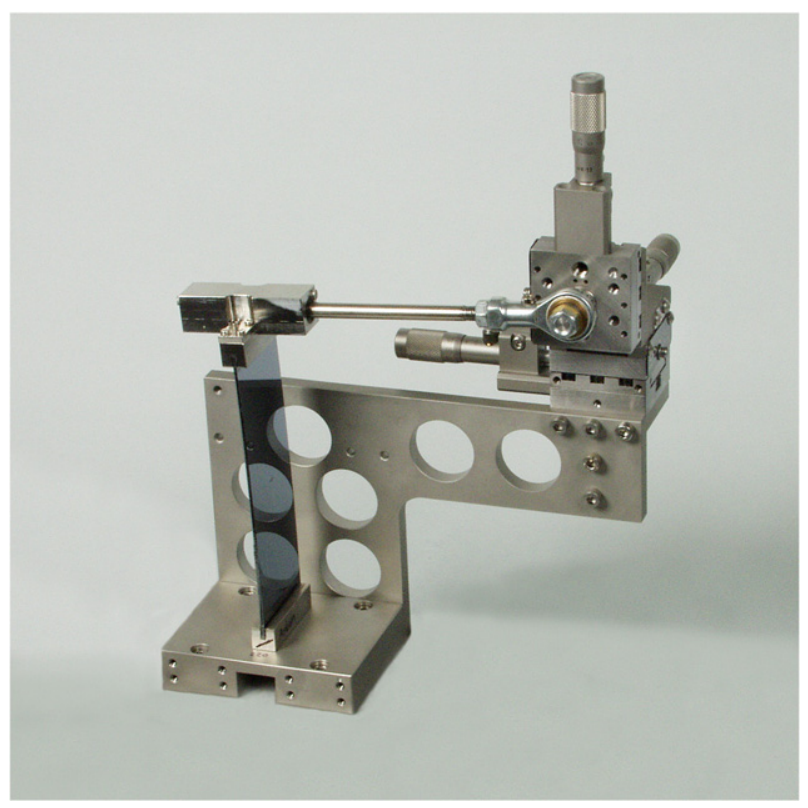

Fig. 7. The silicon crystal mounted in its bending device.
The setup follows the crystal optics explained in Section 3. X-rays emitted from the fast ions are collected by a curved crystal and, for a particular wavelength, Bragg reflection occurs within two small zones on the crystal. For each spectrometer two detector positions are shown which in practice were accessible with a vertically movable detector platform. A cross section taken vertically through one FOCAL spectrometer is displayed in Fig. 6 showing the modular design consisting of the source container for the radioactive probes, the crystal assembly, a connection tube and the detector stage.

\subsection{The crystal bending device}

A silicon plate of dimensions $120 \times 40 \times 1.5 \mathrm{~mm}^{3}$ is mounted and elastically bent in the bending device depicted in Fig. 7 to a radius of curvature amounting to $2 \mathrm{~m}$.

The (220) lattice planes are used for X-ray diffraction in the asymmetric Laue case making an angle of $88^{\circ}$ with the large crystal faces. In choosing an optimum lattice-plane orientation there is still a degree of freedom given by the rotation around the $\langle 110\rangle$ axis. The rotation is selected such as to minimize anticlastic bending [9]. A calculated maximum anticlastic radius of $30 \mathrm{~m}$ is obtained when the $\langle 1 \overline{1} 0\rangle$ axis is aligned with the principal surfaces. Such a large anticlastic radius is of little concern at low Bragg angles in the Laue case.

The radius of curvature can be adjusted by a micrometer screw acting on the sliding lever arm seen in Fig. 7. In addition, the crystal twist can be controlled by another micrometer screw. During the bending procedure the curvature and twist are monitored by observing laser-light reflected from one of the polished surfaces. 

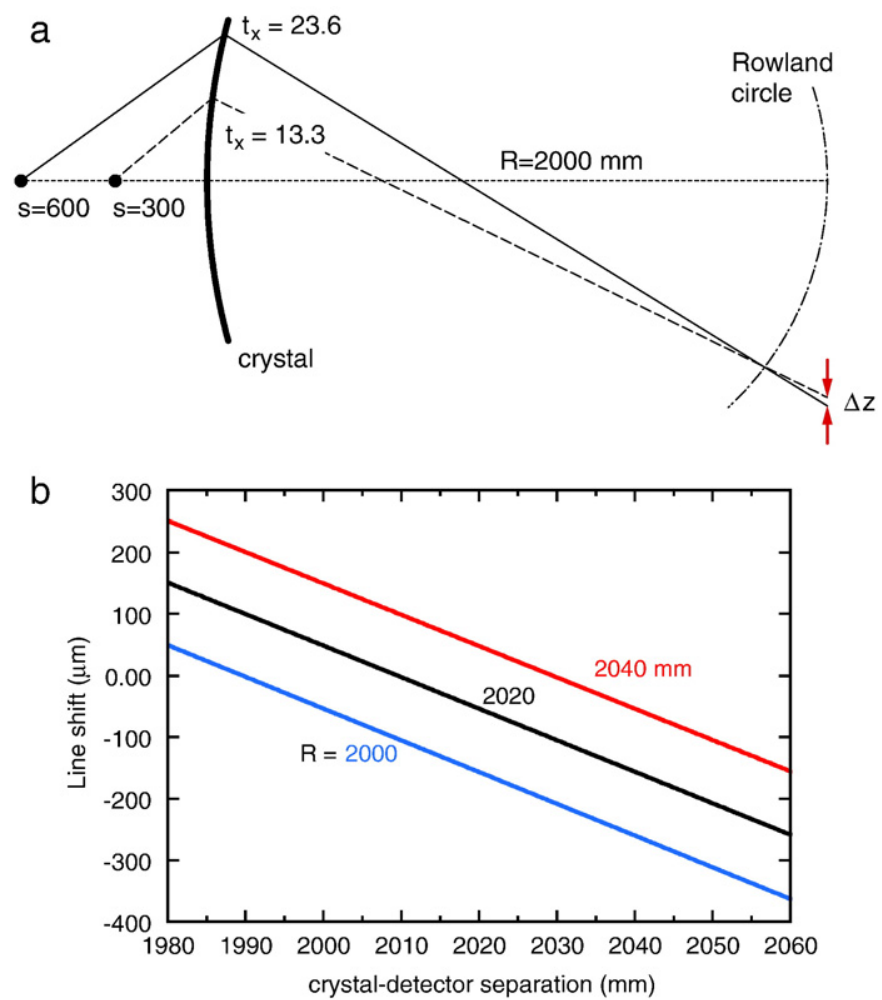

Fig. 8. A small line shift is caused by the change of the source position depending on the position of the detector and on the radius of curvature $R$ of the crystal.

\subsection{Source offset correction}

In the FOCAL X-ray optics-as outlined in Section 3-the locus of the $\mathrm{X}$-ray image on the Rowland circle stays remarkably constant when the source position fluctuates. Only when the source position changes by large amounts two consequences have to be considered: first the position of the polychromatic focus moves and secondly, deviations of the detector location from the Rowland circle become important. A shift of the polychromatic focus can be easily accommodated by simply adjusting any shielding material introduced.

The second issue is more difficult to deal with because it necessitates an accurate knowledge of the detector's position relative to the Rowland circle requiring both a good knowledge of the crystal's bending radius and an accurate method of detector alignment.

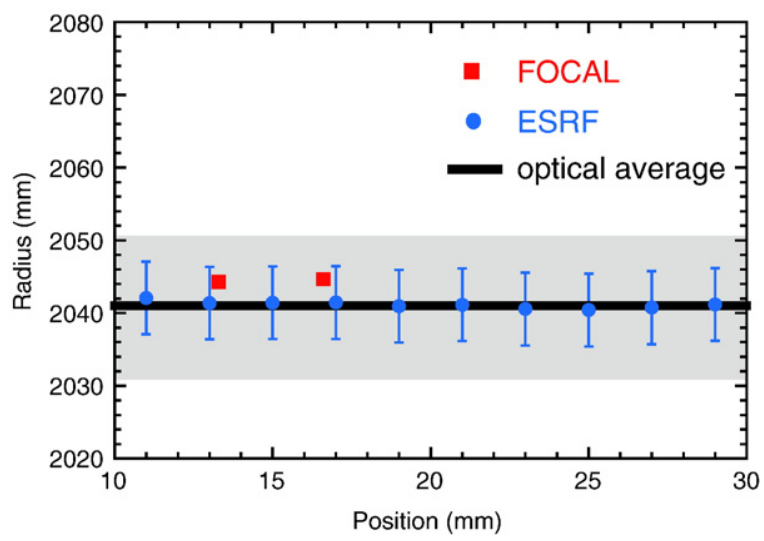

Fig. 9. The radius of curvature of the silicon crystal as a function of the vertical position on the crystal as measured from the centre of the crystal and shown only for the relevant portion of the 120-mm-long crystal. Three different methods were used: FOCAL in scanning mode, synchrotron radiation at the ESRF and optical reflection from the polished crystal surface.

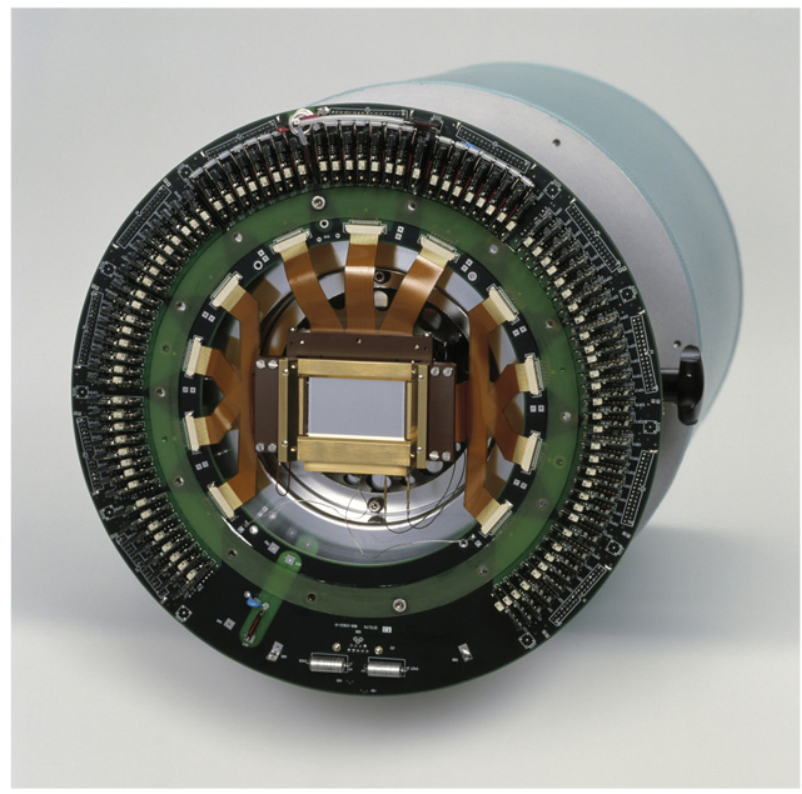

Fig. 10. Photograph of the 2D position-sensitive germanium detector with the front cover opened. The Ge crystal seen in the centre is surrounded by the preamplifier boards connected to the individual strips of the crystal [10].

The upper part of Fig. 8 contains a ray diagram for two largely different source positions. The example is for the present situation where the fast-beam source is at a distance $s$ of $600 \mathrm{~mm}$ from the crystal and the calibration source is at only $300 \mathrm{~mm}$. As a consequence there is a systematic line shift $\Delta z$ calculated for three different radii of curvature as a function of the distance of the detector plane from the crystal. As can be deduced from the graph, the sensitivity on both the detector location and on the radius of curvature amounts to about $5 \mu \mathrm{m} / \mathrm{mm}$.

The curves of Fig. 8 can serve for the necessary crystal-optical correction when dealing with large source offsets. Of course the accuracy of this correction depends on the accuracy to which the crystal's curvature is known.

\subsection{The radius of curvature}

During the course of preparing the present experimental tests three different methods were employed characterizing the curvature of the crystals. First, optical reflections on the polished surfaces were observed from a twin laser beam across which the crystal was scanned in the vertical direction. From this measurement a mean curvature was gained averaged over the centre $80 \mathrm{~mm}$ along the large crystal dimension. Secondly, using synchrotron radiation a more detailed

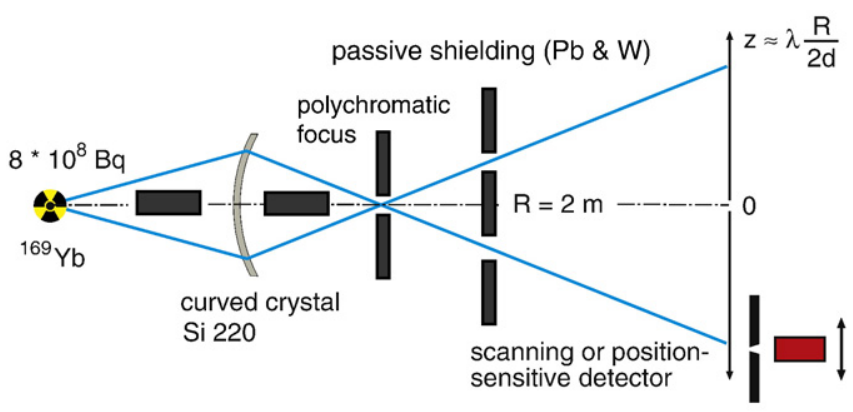

active $\Delta \mathrm{t}, \Delta \mathrm{E}$

Fig. 11. Passive and active shielding making use of the crystal optics with a polychromatic focus and of the time $(\Delta t)$ and energy $(\Delta E)$ resolving capabilities of the X-ray detector. 
curvature test was made scanning the same region with a well collimated $60 \mathrm{keV}$ X-ray beam from the ESRF in Grenoble. Thirdly, FOCAL delivered two distinct measurement points when used in scanning mode with a narrow slit and accessing lower and upper reflections for two different emission lines of ${ }^{169} \mathrm{Yb}$ with known X-ray energies near 50 and $63 \mathrm{keV}$, respectively.

In Fig. 9 the radius of curvature derived from the three methods is plotted for the region of the crystal relevant for the spectroscopic studies. The estimated uncertainties of the methods differ. The optical average is assumed to be accurate to $\pm 10 \mathrm{~mm}$, the measurement points taken at the ESRF have an estimated uncertainty of $\pm 5 \mathrm{~mm}$ and the two single points taken with FOCAL are estimated to lie in a $\pm 1 \mathrm{~mm}$ confidence range. Within their uncertainties all three methods are compatible.

\subsection{The position-sensitive $X$-ray detector}

A two-dimensionally position-sensitive germanium strip detector has been developed to fit the needs of the present application [10]. A photograph of the opened detector is displayed in Fig. 10 showing, in the centre, the Ge crystal surrounded by the electrical connections to the individual preamplifier boards arranged radially on an outer circle. The 11-mm-thick Ge crystal has an active area of $56 \mathrm{~mm} \times 32 \mathrm{~mm}$ and a 128-strip structure with a pitch of $250 \mu \mathrm{m}$ etched on the front surface and a 48-strip structure with a pitch of $1167 \mu \mathrm{m}$ etched on the rear. The finer structure was used to give a higher position resolution in the vertical i.e. in the dispersive direction of the spectrometer.

This detector was mounted on the detector platform of one of the two spectrometers. The other spectrometer was equipped with a previously built prototype detector that has most of the features of the new detector but has position resolution only in one direction [11].
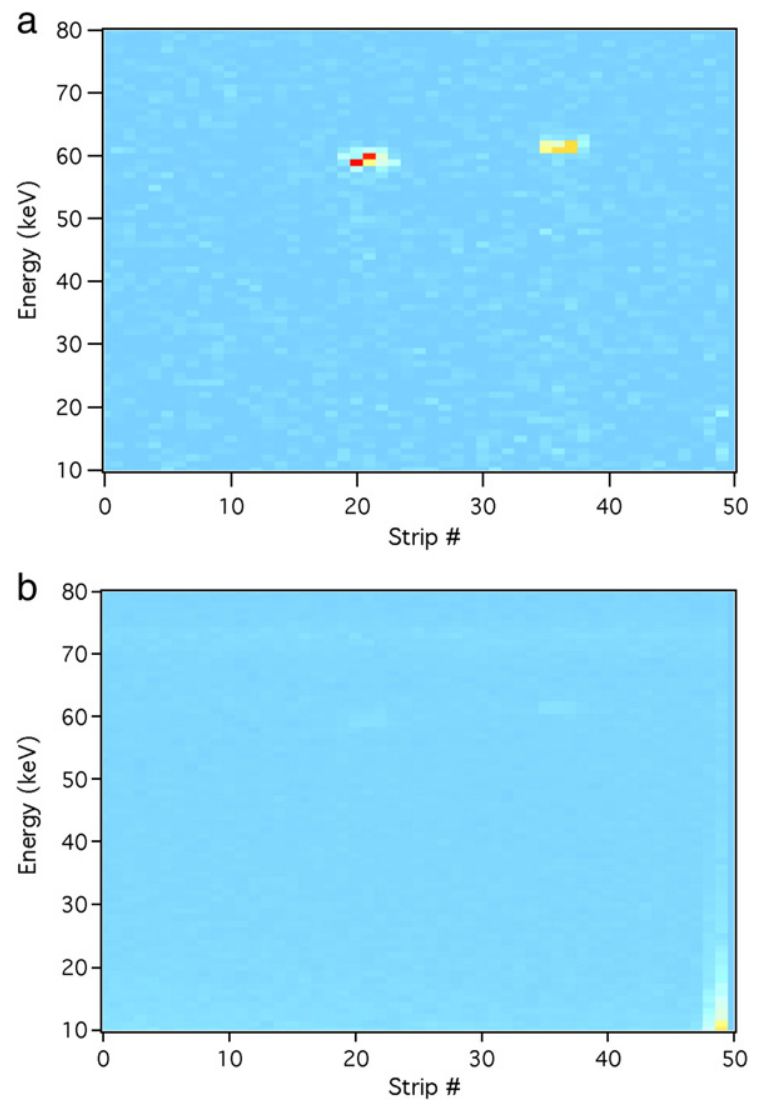

Fig. 12. False-color plot of the X-ray intensity measured with the one-dimensional position-sensitive detector with (top) and without (bottom) time condition. Shown is the intensity as a function of the energy as derived from the signal pulse height and of the strip number which is proportional to the X-ray wavelength. The two spots are due to the Lyman- $\alpha_{1,2}$ doublet of $\mathrm{Pb}^{81+}$.

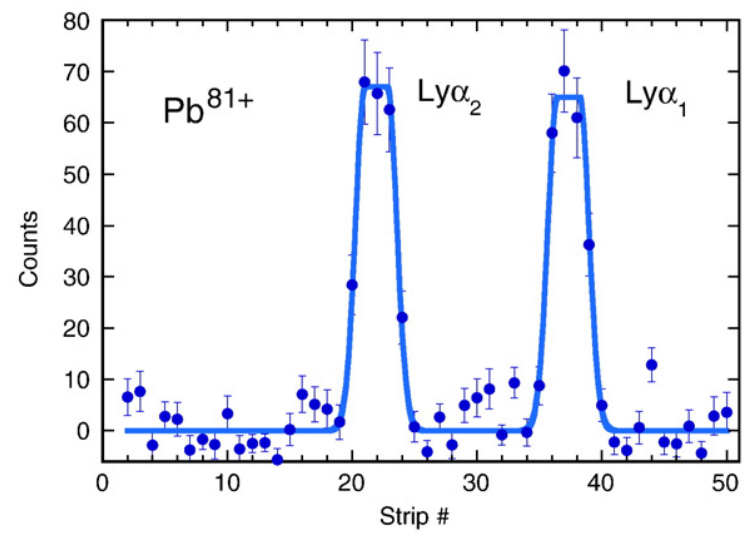

Fig. 13. Intensity profile obtained by integrating the measured intensity of Fig. 12 over an appropriate interval of the energy as derived from the pulse height of the detector. The data are fitted with a top-flat double peak resembling a rotation of the spectral lines out of the horizontal orientation which remains unresolved with a detector that is position-sensitive only in the vertical direction.

Also the active width of the one-dimensional detector is only $23.4 \mathrm{~mm}$ in place of $56 \mathrm{~mm}$ for the new one.

\subsection{Background suppression}

Because the count rates are very low and the spectrometers are operated in an accelerator environment with copious potential X-ray and gamma-ray background it is important to reduce the background as much as possible.

Fig. 11 illustrates the shielding methods used. Passive shielding by $\mathrm{Pb}$ and $\mathrm{W}$ can be readily applied and is facilitated by the existence of a polychromatic focus. Only X-rays originating from the X-ray source of interest diffracted at the proper region of the crystal and passing a 6$\mathrm{mm}$ wide slit located at the polychromatic focus can hit the detector. This slit, although unimportant for the working of the X-ray optics, limits the depth of sight of the instrument to only a few millimeters. Because the position of the polychromatic focus changes when switching between calibration and fast-beam source the $10-\mathrm{mm}$ thick slit jaws made of W alloy were mounted on a rail and so could be remotely shifted. In addition the whole spectrometer has a $15-\mathrm{mm}-$ thick lead cover, as indicated in Fig. 6, preventing radiation from the outer environment from entering the spectrometer.

Furthermore active shielding is employed by making use of the detectors' timing and energy resolving capabilities. Narrow time and energy windows set by software enable us to view only the interesting cuts through the multidimensional measurement data.

\section{Results}

\section{1. $1 D$ detector}

Fig. 12 is a demonstration of the effective background suppression through an X-ray-particle coincidence.

Displayed is a false-color plot of the accumulated intensity as a function of the strip number, which is proportional to the photon wavelength, and as a function of the energy derived from the charge amplitude. Only when a software time window of approximately 100 ns is in effect, as in the top of Fig. 12, a high contrast is achieved.

From the latter an intensity profile of the Lyman- $\alpha_{1,2}$ doublet of $\mathrm{Pb}^{81+}$ can be generated, as shown in Fig. 13, by performing an integration over the X-ray energy in the region of interest.

The measured data are consistent with a top-flat double peak shown as the continuous curve in Fig. 13. Such a profile is expected when the spectral lines are rotated out of the horizontal plane due to the Doppler effect. This phenomenon will be addressed in greater detail in the next 

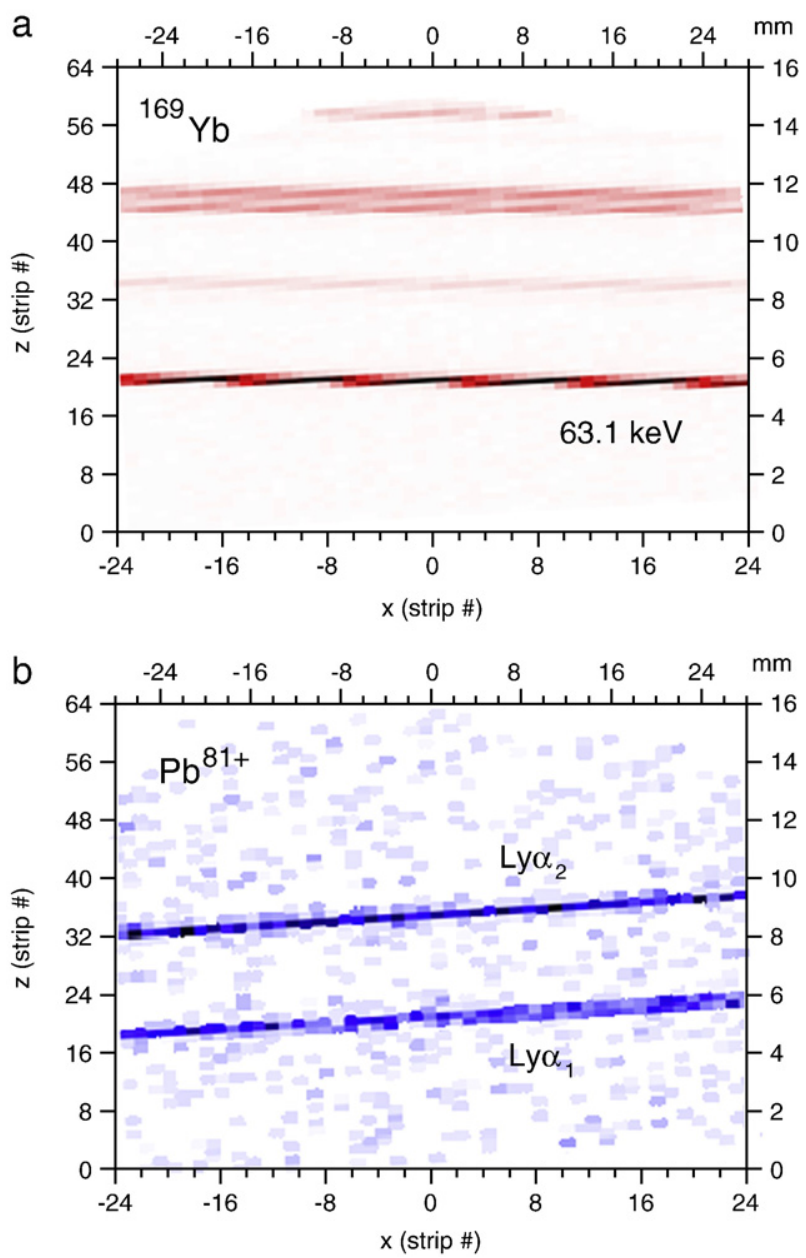

Fig. 14. Two-dimensional image recorded for the (top) stationary calibration source with the prominent 63-keV line and for the (bottom) $\mathrm{Pb}^{81+}$ Lyman- $\alpha$ doublet of the fast-beam source, respectively.

section. Note that the detector's strips are aligned horizontally. In comparison, measured profiles of the $63-\mathrm{keV}$ calibration line emitted by the stationary source reveal a much smaller width.

\section{2. $2 \mathrm{D}$ detector}

During the same measurement period of 10 days, data were collected with the new two-dimensional strip detector at a net count rate of about

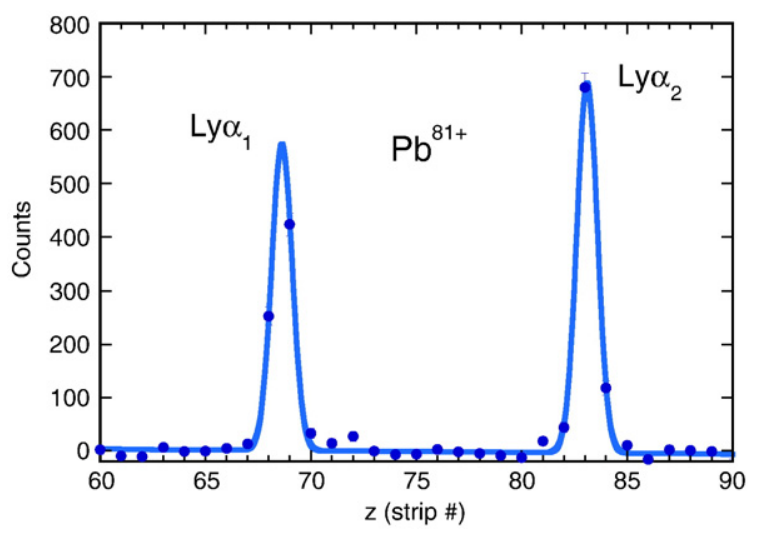

Fig. 15. Spectrum of the Lyman- $\alpha$ doublet obtained by a projection of the measured twodimensional image along the slanted lines.
Table 1

Doppler slanting: predicted and observed slopes of the Lyman- $\alpha_{1,2}$ spectral lines of $\mathrm{Pb}^{81+}$ at a velocity of $\beta \equiv v / c=0.5865$.

\begin{tabular}{lll}
\hline & Lyman- $\alpha_{1}$ & Lyman- $\alpha_{2}$ \\
\hline Predicted & 0.0232 & 0.0240 \\
Observed & $0.0226(6)$ & $0.0237(6)$ \\
\hline
\end{tabular}

10 counts per hour. Timing and energy resolving capabilities were employed in a similar fashion as for the one-dimensional detector.

As an end result Fig. 14 shows the accumulated coincident twodimensional image of the $\mathrm{Pb}$ Lyman- $\alpha$ lines in comparison with that of the stationary ${ }^{169} \mathrm{Yb}$ source. Please note that the pitch in the vertical direction is by a factor of about 4.7 smaller than in the horizontal direction. As opposed to lines from the stationary source, the spectral lines from the fast $\mathrm{Pb}$ beam show a slope due to the Doppler effect. It is conceivable that the resolution deteriorates if the slanting remains unresolved. From the 2D image an intensity profile is obtained by a projection along the slanted lines which is depicted in Fig. 15.

The width of the impinging intensity distribution, along the vertical direction, cannot be accurately determined because it is only in the order of one half of the detector's pitch of $250 \mu \mathrm{m}$, also in accordance with our ray-tracing simulations. Employing a proper twodimensional image analysis, the line position can be extracted quite accurately. In the present configuration, the 2D intensity distribution can be located with an estimated uncertainty of $\pm 6 \mu \mathrm{m}$ in the dispersive direction mainly limited by the low counting statistics. It turned out that the mechanical stability of our detector platform does not match this value. Vertical excursions in the detector position were provoked by the periodic change in detector weight due to regular evaporation of liquid nitrogen followed by refilling the detectors' dewars.

Table 1 compares the measured slopes of the Pb Lyman- $\alpha$ lines to a calculation performed with MacRay. A good agreement within the estimated measurement uncertainty can be stated. There also is a small, though experimentally insignificant, difference in the slopes of the two lines according to their wavelength difference.

\section{Conclusion}

The FOCAL X-ray optics have been developed for spectroscopy in a demanding situation. Through careful optimization of the relevant parameters and implementation into a twin spectrometer it was possible to demonstrate the ability to perform measurements on the rather dim source of X-rays emitted in flight from one-electron lead ions. With the new 2D position-sensitive X-ray detector it was possible to quantitatively verify the Doppler slanting. X-ray optical corrections necessary in the calibration process were identified. In the future we will try to largely avoid them by installing a new positioning mechanism allowing us to place the calibration source near the position of the fastbeam source.

A second two-dimensionally position-sensitive X-ray detector is presently under construction. Having both spectrometers equipped with a 2D detector will enhance measurement speed and wavelength accuracy along with robustness towards possible fluctuations in the direction of the fast ion beam.

\section{Acknowledgements}

The present experiments were only possible with the excellent ion beams prepared by the GSI accelerator crew and especially by the experts of the ESR. We thank W. Brüchle for preparing the radioactive samples and B. Lommel, B. Kindler and the crew of the GSI target lab for assistance in gluing the crystals. The professional help from J.P. Vassalli of the ESRF crystal lab and from A. Rommeveaux of the ESRF 
Optics Metrology lab is thankfully acknowledged. We thank T. Bigault and R. Hustache for their kind assistance at the optics beamline of the ESRF. We thank W. Enders and H. Wesp for their technical assistance during spectrometer assembly. This research has been supported by a Marie Curie Fellowship of the European Community Programme IHP under contract number HPMT-CT-2000-00197. The financial support of the Swiss National Science Foundation is acknowledged.

\section{References}

[1] Th. Stöhlker, H.F. Beyer, A Gumberidze, A Kumar, D. Liesen, R. Reuschl, U. Spillmann, M. Trasinelli, Ground-state Lamb shift of heavy hydrogen-like ions: status and perspectives, Hyperfine Interact. 172 (2006) 135-140.

[2] H.F. Beyer, FOCAL: X-ray optics for accurate spectroscopy, Spectrochim. Acta Part B 59 (2004) 1535-1542.

[3] V. Andrianov, K. Beckert, P. Beller, A. Bleile, P. Egelhof, A. Gumberidze, S. Ilieva, C. Kilbourne, H.J. Kluge, S. Kraft-Bermuth, D. McCammon, J.P. Meier, U. Popp, R. Reuschl, T. Stöhlker, S. Trotsenko, First observation of the Lyman- $\alpha$ transition in hydrogen-like heavy ions with high-resolution calorimetric low-temperature detectors, in: GSI Scientific Report 2005, GSI Report 2006-1, ISSN: 0174-0814, 2006, p. 299.
[4] H.F. Beyer, Introduction to the Physics of Highly Charged Ions, IOP, Bristol, 2003.

[5] H.F. Beyer, Characterization of transmission-type curved-crystal X-ray optics for fast ion-beam spectroscopy, Nucl. Instrum. Methods A400 (1997) 137-148.

[6] P. Penning, Anomalous transmission of x rays in elastically deformed crystals, Philips Res. Rep. (1961) 419-440.

[7] E. Erola, X-ray reflectivity of bent perfect crystals in Bragg and Laue geometry, J. Appl. Cryst. (1990) 35-42.

[8] M. Sánchez del Río, R.J. Dejus, Status of XOP: an X-ray optics software toolkit, Proceedings SPIE 5536 (2004) 171-174.

[9] M. Krisch, Study of dynamically bent crystals for X-ray focusing optics, Nucl. Instrum. Methods. 308 (1991) 378-381.

[10] D. Protić, T. Stöhlker, T. Krings, I. Mohos, U. Spillmann, Two-dimensional microstrip germanium detector for the spectroscopy of hard X-ray transitions, IEEE Trans. Instr. Meas. 52 (2005) 3194-3198;

Th. Stöhlker, U. Spillmann, D. Banas, H.F. Beyer, J.-Cl. Dousse, S. Chatterjee, S. Heß, Chr. Kozhuharov, M. Kavčič, T. Krings, D Protić, R. Reuschl, J. Szlachetko, S. Tashenov, S. Trotsenko, A 2D position sensitive germanium detector for spectroscopy and polarimetry of high-energetic X-rays, J. Phys. Conference Ser. 58 (2007) 411-414.

[11] D. Protić, T. Stöhlker, H.F. Beyer, J. Bojowald, G. Borchert, A.Gumberidze, A. Hammacher, C. Kozhuharov, X. Ma, I. Mohos, Amicro-strip germanium detector for position sensitive X-ray spectroscopy, IEEE Trans. Instr. Meas. 48 (2001) 10481052. 